\title{
THE MOMENT PROBLEM FOR SOME WIENER FUNCTIONALS: CORRECTIONS TO PREVIOUS PROOFS (WITH AN APPENDIX BY H. L. PEDERSEN)
}

\author{
PER HÖRFELT, ${ }^{*}$ Chalmers University of Technology
}

\begin{abstract}
In this paper, we describe a class of Wiener functionals that are 'indeterminate by their moments', that is, whose distributions are not uniquely determined by their moments. In particular, it is proved that the integral of a geometric Brownian motion is indeterminate by its moments and, moreover, shown that previous proofs of this result are incorrect. The main result of this paper is based on geometric inequalities in Gauss space and on a generalization of the Krein criterion due to H. L. Pedersen.
\end{abstract}

Keywords: Wiener functional; moment problem; geometric inequality

2000 Mathematics Subject Classification: Primary 44A60; 60J65

\section{Introduction}

Suppose that $\left\{W_{t}\right\}_{0 \leq t \leq 1}$ is a standard one-dimensional Brownian motion on $[0,1], \theta \in \mathbb{R}$, and that $\sigma>0$. In [16], Yor studied the question of whether the random variable

$$
\int_{0}^{1} \mathrm{e}^{\theta t+\sigma W_{t}} \mathrm{~d} t
$$

is 'determinate by its moments', that is, if the law of the random variable is uniquely determined by its moments. Nikeghbali, and later on also the author, showed that a result of Pakes [12] implied that the integral of a geometric Brownian motion is 'indeterminate by its moments' (see Nikeghbali [11] and Hörfelt [8]). However, the result by Pakes turned out to be false, as recently shown in [3] and, thus, the proofs of the results about indeterminacy in [11] and [8] have to be re-examined.

This paper will prove that the integral of a geometric Brownian motion is indeed indeterminate by its moments. In addition, the main result of this paper implies that other Wiener functionals are indeterminate by their moments. For instance, suppose that $\mu$ is a positive bounded Borel measure on $[0,1]$, with $\mu((0,1])>0$ and $p>4$. Then the random variable

$$
\int_{0}^{1}\left|\theta t+\sigma W_{t}\right|^{p} \mu(\mathrm{d} t)
$$

is indeterminate by its moments. This result may be compared with a classic result by Berg stating that the random variable $\left|W_{1}\right|^{p}, p>0$, is determinate by its moments if and only if $p>4$ (see Berg [1]). We will give the exact statement of our result in Section 2.

Received 3 November 2003; revision received 11 February 2005.

* Postal address: Fraunhofer-Chalmers Research Centre for Industrial Mathematics, Chalmers Science Park, SE-412 88 Göteborg, Sweden. Email address: perh@fcc.chalmers.se

The author would like to thank Christer Borell, Chalmers University of Technology. 
The new proof will be based on a generalization of the Krein condition due to Pedersen [13] and some geometric inequalities in Gauss space.

The paper is structured as follows. In Section 2, we introduce some notation and present the main result. In Section 3, we discuss geometric bounds on the distribution functions for a class of Wiener functionals. In Section 4, we present an extension of the Krein criterion for determinacy and, in Section 5, we prove the main result. We conclude the paper with an appendix by Pedersen. This appendix gives a relatively simple and self-contained proof of the main result in Section 4.

\section{Notation and main result}

From now on, the sample space $\Omega=C_{0}\left([0,1] ; \mathbb{R}^{n}\right)$ consists of all functions $\omega=$ $\left(\omega_{1}, \omega_{2}, \ldots, \omega_{n}\right)$ such that, for each $i=1, \ldots, n$, the function $\omega_{i}:[0,1] \rightarrow \mathbb{R}$ is continuous and $\omega_{i}(0)=0$. The space $\Omega$ is equipped with the norm $\|\cdot\|_{C_{0}}$, defined by

$$
\|\omega\|_{C_{0}}=\max _{i=1, \ldots, n} \max _{0 \leq t \leq 1}\left|\omega_{i}(t)\right| .
$$

The measure $P$ will henceforth denote the Wiener measure on $\Omega$. In particular, if we define

$$
W_{t}(\omega)=\omega_{1}(t), \quad 0 \leq t \leq 1,
$$

then $\left\{W_{t}\right\}_{0 \leq t \leq 1}$ is a standard Brownian motion on $[0,1]$ with respect to $P$.

Let $I$ be an interval with closure $[0, \infty)$, and suppose that $\psi: I \rightarrow \mathbb{R}$ is a continuous function such that $\psi(\infty)=\infty$. Furthermore, suppose that $\psi$ is differentiable on the interior of $I$ and that $\psi^{\prime}(s)>0$ for all $s>0$. Next, consider a function $\Psi: \Omega \rightarrow(0, \infty)$. We will say that $\Psi \in \mathcal{L}(\psi)$ if the map $\omega \mapsto \psi \circ \Psi(\omega)$ is $C_{0}$-Lipschitz continuous with constant 1 . That is, $\Psi \in \mathcal{L}(\psi)$ if

$$
|\psi \circ \Psi(\omega+\tilde{\omega})-\psi \circ \Psi(\omega)| \leq\|\tilde{\omega}\|_{C_{0}},
$$

for all $\omega, \tilde{\omega} \in \Omega$. Furthermore, we write $\Psi \in \mathcal{C}(\psi)$ if the map $\omega \mapsto \psi \circ \Psi(\omega)$ is convex. Thus, $\Psi \in \mathcal{C}(\psi)$ if

$$
\psi \circ \Psi(\lambda \omega+(1-\lambda) \tilde{\omega}) \leq \lambda \psi \circ \Psi(\omega)+(1-\lambda) \psi \circ \Psi(\tilde{\omega}),
$$

for all $0 \leq \lambda \leq 1$ and all $\omega, \tilde{\omega} \in \Omega$.

Next we give some examples of functionals in $\mathcal{L}(\psi)$ and $\mathcal{C}(\psi)$. If $p \geq 1, \theta \in \mathbb{R}, \sigma>0$, and

$$
\Psi(\omega)=\int_{0}^{1}\left|\theta t+\sigma \omega_{1}(t)\right|^{p} \mu(\mathrm{d} t),
$$

where $\mu$ is a positive, bounded Borel measure on $[0,1]$ with $\mu((0,1])>0$, then the Minkowski inequality gives $\Psi \in \mathcal{L}(\psi) \cap \mathcal{C}(\psi)$, where

$$
\psi(s)=\frac{1}{\sigma \mu([0,1])} s^{1 / p} .
$$

Suppose that $\mu$ is defined as above, that $\theta_{i} \in \mathbb{R}$ and $\sigma_{i}>0, i=1, \ldots, n$, and define

$$
\Psi(\omega)=\int_{0}^{1} \sum_{i=1}^{n} \mathrm{e}^{\theta_{i} t+\sigma_{i} \omega_{i}(t)} \mu(\mathrm{d} t) .
$$


Then $\Psi \in \mathcal{L}(\psi) \cap \mathcal{C}(\psi)$ with

$$
\psi(s)=\frac{1}{\max _{i=1, \ldots, n} \sigma_{i}} \ln s .
$$

More examples of functionals in $\mathcal{L}(\psi) \cap \mathcal{C}(\psi)$ with $\psi$ as in (3) were described by the author in [8].

Before we give the main result of the paper, recall that a distribution function $F$ with support on the positive real numbers and with moments of all order is Stieltjes indeterminate if there is a distribution function $G$ with support on the positive real numbers such that $G \neq F$ and $\int_{0}^{\infty} s^{k} \mathrm{~d} G(s)=\int_{0}^{\infty} s^{k} \mathrm{~d} F(s)$, for all integers $k$. Moreover, a positive random variable or a probability measure with support on the positive real numbers is Stieltjes indeterminate if the corresponding distribution function is Stieltjes indeterminate.

The purpose of this paper is to prove the following theorem.

Theorem 1. Suppose that $\Psi \in \mathcal{L}(\psi) \cap \mathcal{C}(\psi)$ has moments of all orders and is nonconstant. If

$$
\int_{a}^{\infty} s^{-3 / 2}\left(\psi^{2}(s)+\left|\ln \left(\psi^{\prime}(s)\right)\right|\right) \mathrm{d} s<\infty,
$$

for some $a>0$, then $\Psi$ is Stieltjes indeterminate.

Note that Theorem 1 implies that the functional $\Psi$ in (1) is Stieltjes indeterminate if $p>4$ and that the functional in (2) is Stieltjes indeterminate for all parameter values.

\section{Geometric bounds on the distribution function}

In this section, we consider different bounds on the distribution function of $\Psi$. The distribution function will be denoted by $F_{\Psi}$, i.e.

$$
F_{\Psi}(s)=\mathrm{P}(\Psi \leq s), \quad s \geq 0 .
$$

It is well known that the topological support of $\mathrm{P}$ is $\Omega$. Thus, if $\Psi \in \mathcal{C}(\psi) \cap \mathcal{L}(\psi)$ and $F_{\psi \circ \Psi}\left(s_{0}\right)=1$, then the set $\left\{\omega \in \Omega: \psi \circ \Psi(\omega)>s_{0}\right\}$ is an open zero set and must therefore be empty. Since $\psi \circ \Psi$ is convex, it follows that $\psi \circ \Psi$ is constant. Thus, if $\Psi$ is nonconstant and $\Psi \in \mathcal{C}(\psi) \cap \mathcal{L}(\psi)$ then $F_{\Psi}(s)<1$ for all $s \geq 0$.

Henceforth, we adopt the convention that $\infty-\infty=-\infty$. Moreover, we define

$$
\Phi(x)=\int_{-\infty}^{x} \mathrm{e}^{-y^{2} / 2} \frac{\mathrm{d} y}{\sqrt{2 \pi}}, \quad-\infty \leq x \leq \infty,
$$

and let $\Phi^{-1}$ denote the inverse of $\Phi$. Finally, assume that

$$
\mathrm{P}_{*}(A)=\sup \{\mathrm{P}(K): K \text { is a compact subset of } A\},
$$

for any set $A \subset \Omega$.

The following theorem is a special case of the Ehrhard inequality.

Theorem 2. Suppose that $A$ and $B$ are Borel sets in $\Omega$. Then

$$
\Phi^{-1}\left(\mathrm{P}_{*}(\lambda A+(1-\lambda) B)\right) \geq \lambda \Phi^{-1}(\mathrm{P}(A))+(1-\lambda) \Phi^{-1}(\mathrm{P}(B)),
$$

for every $0 \leq \lambda \leq 1$. 
In [6], Ehrhard proved Theorem 2 for convex sets $A$ and $B$. The general case was shown recently by Borell in [5]. The formulation of the Ehrhard inequality is taken from [5]. In our case, it is possible to replace $\mathrm{P}_{*}$ by $\mathrm{P}$ since $C_{0}$ is a polish space and, therefore, the convex sum of two Borel sets is Borel measurable.

The proof of the following lemma is based on the Ehrhard inequality as well as an idea in [7].

Lemma 1. Suppose that $\Psi \in \mathcal{C}(\psi)$ is nonconstant and continuous, and let $R_{\psi}$ denote the range of $\psi$. The map

$$
x \mapsto \Phi^{-1} \circ F_{\Psi} \circ \psi^{-1}(x), \quad x \in R_{\psi},
$$

is concave. In particular, $F_{\Psi}$ is absolutely continuous on $\left(s_{*}, \infty\right)$, where

$$
s_{*}=\inf \left\{s \geq 0: F_{\Psi}(s)>0\right\} .
$$

Proof. Put $\Upsilon=\psi \circ \Psi$. By the convexity of $\Upsilon$, we obtain

$$
\{\Upsilon \leq \lambda x+(1-\lambda) y\} \supseteq \lambda\{\Upsilon \leq x\}+(1-\lambda)\{\Upsilon \leq y\},
$$

for all $x, y \in \mathbb{R}$ and all $\lambda, 0 \leq \lambda \leq 1$. The Ehrhard inequality implies that

$$
\begin{aligned}
& \Phi^{-1}(\mathrm{P}(\Upsilon \leq \lambda x+(1-\lambda) y)) \\
& \quad \geq \lambda \Phi^{-1}(\mathrm{P}(\Upsilon \leq x))+(1-\lambda) \Phi^{-1}(\mathrm{P}(\Upsilon \leq y))
\end{aligned}
$$

and, hence, $\Phi^{-1} \circ F_{\Psi} \circ \psi^{-1}$ is concave.

This result gives a concave function $g:\left(x_{*}, \infty\right) \rightarrow \mathbb{R}, x_{*}=\psi\left(s_{*}\right)$, such that

$$
F_{\Psi}(s)=\Phi \circ g \circ \psi(s),
$$

for all $s>s_{*}$. A concave function is absolutely continuous and, thus, $g \circ \psi(s), s>s_{*}$, is absolutely continuous. Since $\Phi$ is absolutely continuous, the proof is complete.

From now on, the space $\mathcal{H}$ consists of all functions $h=\left(h_{1}, h_{2}, \ldots, h_{n}\right)$ such that, for each $i=1, \ldots, n$, the function $h_{i}:[0,1] \rightarrow \mathbb{R}$ is absolutely continuous with a square-integrable derivative, and $h_{i}(0)=0$. The space $\mathscr{H}$ is equipped with the norm $\|\cdot\|_{\mathscr{H}}$, defined by

$$
\|h\|_{\mathscr{H}}=\left(\sum_{i=1}^{n} \int_{0}^{1}\left(h_{i}^{\prime}(t)\right)^{2} \mathrm{~d} t\right)^{1 / 2}, \quad h \in \mathcal{H},
$$

where a prime denotes weak-sense differentiation. The space $\mathscr{H}$ is usually referred to as the Cameron-Martin space.

Theorem 3. Suppose that $O_{\mathscr{H}}$ is the set of all $h \in \mathcal{H}$ such that $\|h\|_{\mathcal{H}} \leq 1$, and that $A$ is a Borel set in $\Omega$. If an a is chosen such that

$$
\mathrm{P}(A)=\Phi(a),
$$

then

$$
\mathrm{P}\left(A+\lambda O_{\mathscr{H}}\right) \geq \Phi(a+\lambda)
$$

for each $\lambda \geq 0$.

Theorem 3 is a special case of the celebrated isoperimetric inequality for Gaussian measures, which was discovered independently by Borell [4] and Sudakov and Cirel’son [15]. 
Lemma 2. Suppose that $\Psi \in \mathscr{L}(\psi) \cap \mathcal{C}(\psi)$ is nonconstant and assume that

$$
s_{*}=\inf \left\{s \geq 0: F_{\Psi}(s)>0\right\} .
$$

Then

$$
f_{\Psi}(s) \geq\left(\varphi \circ \Phi^{-1} \circ F_{\Psi}\right)(s) \psi^{\prime}(s), \quad s>s_{*},
$$

where $f_{\Psi}=F_{\Psi}^{\prime}$ and $\varphi=\Phi^{\prime}$.

Proof. Suppose that $O_{C_{0}}$ consists of all $\omega \in \Omega$ such that $\|\omega\|_{C_{0}} \leq 1$. Note that the Hölder inequality implies

$$
\begin{aligned}
\|h\|_{C_{0}} & =\max _{i=1, \ldots, n} \max _{0 \leq t \leq 1}\left|\int_{0}^{t} h_{i}^{\prime}(s) \mathrm{d} s\right| \\
& \leq \max _{i=1, \ldots, n} \max _{0 \leq t \leq 1} \sqrt{t}\left(\int_{0}^{t} h_{i}^{\prime}(s)^{2} \mathrm{~d} s\right)^{1 / 2} \\
& \leq\|h\|_{\mathcal{H}},
\end{aligned}
$$

and, thus, $O_{C_{0}} \supset O_{\mathscr{H}}$, where $O_{\mathscr{H}}$ is defined as in Theorem 3 .

Next, suppose that $\Upsilon=\psi \circ \Psi$ and $A=\{\Upsilon \leq s\}$ for some $s>s_{*}$. Since $\Upsilon$ is $C_{0}$-Lipschitz continuous with constant 1 and $O_{C_{0}} \supset O_{\mathscr{H}}$, it follows that

$$
\{\Upsilon \leq s+\lambda\} \supseteq A+\lambda O_{C_{0}} \supseteq A+\lambda O_{\mathscr{H}},
$$

for each $\lambda>0$. Thus, if $a$ satisfies $\mathrm{P}(A)=\Phi(a)$ then Theorem 3 yields

$$
\mathrm{P}(\Upsilon \leq s+\lambda)-\mathrm{P}(\Upsilon \leq s) \geq \Phi(a+\lambda)-\Phi(a)
$$

and, therefore, $f_{\Upsilon}(s) \geq \varphi(a)$, where $f_{\Upsilon}$ is the density function of $F_{\Upsilon}$. Thus, $f_{\Upsilon}(s) \geq$ $\varphi \circ \Phi^{-1} \circ F_{\Upsilon}(s)$. Lemma 2 now follows by the relation $f_{\Psi}(s)=\left(f_{\Upsilon} \circ \psi\right)(s) \psi^{\prime}(s)$.

\section{The moment problem}

A famous result in the theory of the moment problem is the Krein condition. The following theorem presents a generalization of Krein's result established by Pedersen [13]. A relatively simple proof of this result is shown in Appendix A.

Theorem 4. Suppose that $\mathrm{d} \mu(x)=f(x) \mathrm{d} x$ is a probability measure on $[0, \infty)$ with moments of all orders. If, for some $a \geq 0$,

$$
\int_{s \geq a} \frac{\log f\left(s^{2}\right)}{1+s^{2}} \mathrm{~d} s>-\infty,
$$

then $\mu$ is Stieltjes indeterminate.

We will need a slight modification of Theorem 4.

Corollary 1. Suppose that the distribution function $F$ is absolutely continuous on $\left(s_{*}, \infty\right)$, where $s_{*}=\inf \{s \in \mathbb{R}: F(s)>0\} \geq 0$. Assume that $F$ has moments of all orders and that $f(s)=F^{\prime}(s), s>s_{*}$, satisfies

$$
\int_{a}^{\infty} \frac{\ln f\left(s^{2}\right)}{1+s^{2}} \mathrm{~d} s>-\infty,
$$

for some $a>s_{*}$. Then $F$ is Stieltjes indeterminate. 
Proof. Define $C=F\left(s_{*}\right)$. It is evident that $C<1$. Introduce the distribution function

$$
G(s)= \begin{cases}\frac{F\left(s+s_{*}\right)-C}{1-C}, & s \geq 0, \\ 0, & s<0 .\end{cases}
$$

This function is absolutely continuous on $[0, \infty)$ and, by Theorem 4 , Stieltjes indeterminate. Suppose that $H \neq G$ is a distribution function with support on $[0, \infty)$ and with the same moments as $G$. In addition, define

$$
J(s)= \begin{cases}(1-C) H\left(s-s_{*}\right)+C, & s \geq s_{*}, \\ 0, & s<s_{*},\end{cases}
$$

and recall that

$$
F(s)= \begin{cases}(1-C) G\left(s-s_{*}\right)+C, & s \geq s_{*}, \\ 0, & s<s_{*} .\end{cases}
$$

It is evident that $J$ is a distribution function such that $J \neq F$ and $J(s)=0, s<0$. However, $J$ has the same moments as $F$. Thus, $F$ is Stieltjes indeterminate.

\section{Proof of Theorem 1}

Now, to prove the main result, suppose that $\Psi \in \mathcal{L}(\psi) \cap \mathcal{C}(\psi)$ with

$$
\int_{a}^{\infty} s^{-3 / 2}\left(\psi^{2}(s)+\left|\ln \left(\psi^{\prime}(s)\right)\right|\right) \mathrm{d} s<\infty,
$$

for some $a>0$. Define, as previously, $F_{\Psi}(s)=\mathrm{P}(\Psi \leq s)$ and $f_{\Psi}(s)=F_{\Psi}^{\prime}(s)$ if $s>s_{*}=$ $\inf \left\{s \geq 0: F_{\Psi}(s)>0\right\}$. By Theorem $1, \Psi$ is Stieltjes indeterminate if

$$
\int_{a}^{\infty} \frac{\ln f_{\Psi}\left(s^{2}\right)}{1+s^{2}} \mathrm{~d} s>-\infty,
$$

for some $a>s_{*}$, or, equivalently,

$$
\int_{a}^{\infty} s^{-3 / 2} \ln f_{\Psi}(s) \mathrm{d} s>-\infty .
$$

Lemma 2 yields

$$
\int_{a}^{\infty} s^{-3 / 2} \ln f_{\Psi}(s) \mathrm{d} s \geq \int_{a}^{\infty} s^{-3 / 2} \ln \left[\left(\varphi \circ \Phi^{-1} \circ F_{\Psi}\right)(s) \psi^{\prime}(s)\right] \mathrm{d} s .
$$

The right-hand side equals

$$
-\frac{1}{2} \int_{a}^{\infty} s^{-3 / 2}\left(\Phi^{-1} \circ F_{\Psi}(s)\right)^{2} \mathrm{~d} s+\int_{a}^{\infty} s^{-3 / 2} \ln \psi^{\prime}(s) \mathrm{d} s-2 \sqrt{a} \ln (2 \pi) .
$$

The proof of Theorem 1 is thus complete if we can prove that each integral in (5) is finite.

Lemma 1 gives constants $m$ and $k$ such that $\Phi^{-1} \circ F_{\Psi} \circ \psi^{-1}(x) \leq k x+m$, for all $x$ in the range of $\psi$, or, equivalently, $\Phi^{-1} \circ F_{\Psi}(s) \leq k \psi(s)+m$, for all $s>0$. Combined with (4), it follows that the first integral in (5) is finite. Furthermore, (4) gives at once that the integral $\int_{a}^{\infty} s^{-3 / 2} \ln \psi^{\prime}(s) \mathrm{d} s$ is finite. The proof of Theorem 1 is complete. 


\section{Appendix A by H. L. Pedersen: The Krein condition for the moment problem}

The purpose of this appendix is to give a relatively simple proof of Theorem 4 . Theorem 4 can be obtained from the corresponding theorem for measures on the real line, stated as follows.

Theorem 5. Suppose that $\mathrm{d} \mu(x)=h(x) \mathrm{d} x$ is a probability measure on $\mathbb{R}$ having moments of all orders. If, for some $a \geq 0$,

$$
\int_{|x| \geq a} \frac{\log h(x)}{x^{2}+1} \mathrm{~d} x>-\infty
$$

then $\mu$ is indeterminate on $\mathbb{R}$.

This theorem goes back to Krein [10], who obtained it for $a=0$. (For a simple proof in this case see, for example, [2].) It is frequently called 'Krein's condition for indeterminacy'. Theorem 4 is obtained from Theorem 5 by considering the symmetric measure $|x| f\left(x^{2}\right) \mathrm{d} x$ on $\mathbb{R}$, as in [2].

Theorem 5 can be seen as a corollary of Krein's condition for indeterminacy over sets of so-called positive lower uniform density (see [13]). Indeed, the set $\{|x| \geq a\}$ contains a set of this form. The proofs in [13] were based on estimates of harmonic measure due to Carleson and results of Koosis about harmonic estimation (see [9, Chapter VIII]). The point is that Theorem 5 is much more elementary to prove, although the proof below is still based on harmonic estimation. For an introduction to harmonic measures see, for example, [14] and [9].

The harmonic measure $\omega_{\Delta}$ in the unit disk $\Delta$ is defined in terms of the Poisson kernel. We have

$$
\omega_{\Delta}(\Omega, w)=\frac{1}{2 \pi} \int_{\Omega} \frac{1-|w|^{2}}{\left|\mathrm{e}^{\mathrm{i} t}-w\right|^{2}} \mathrm{~d} t,
$$

for any Borel set $\Omega$ in $[-\pi, \pi)$ and any point $w \in \Delta$.

We shall define and use the harmonic measure $\omega_{\mathcal{D}}$ in the simply connected domain

$$
\mathscr{D}=\mathbb{C} \backslash((-\infty,-1] \cup[1, \infty)),
$$

by using a conformal mapping of $\mathscr{D}$ onto $\Delta$.

It is easily checked that $\psi(w)=1 /((w+1 / w) / 2)$ is a conformal mapping of $\Delta$ onto $D$ and, hence, that $\phi(z)=1 / z-\left(1 / z^{2}-1\right)^{1 / 2}$ is a conformal mapping of $\mathscr{D}$ onto $\Delta$ with $\phi(0)=0$ (for the square root in $\phi(z)$ that is positive for $z \in(-1,1)$ ).

We consider the boundary of $\mathcal{D}$ to have upper and lower sides (see, for example, [9, Chapter VIII, A.1]), due to the fact that $\psi$ maps the unit circle onto $\partial \mathscr{D}$ in a two-to-one way $\left(\psi\left(\mathrm{e}^{\mathrm{i} \theta}\right)=1 / \cos \theta\right)$. Hence, we think of a Borel set $E$ in $\partial \mathscr{D}$ as having two sides $E_{+}$and $E_{-}$. For $x \in \mathbb{R}$, we put

$$
\phi_{+}(x)=\lim _{y \rightarrow 0+} \phi(x+\mathrm{i} y), \quad \phi_{-}(x)=\lim _{y \rightarrow 0-} \phi(x+\mathrm{i} y) .
$$

It makes sense to talk about $t w o$ harmonic measures, namely $\omega_{\mathcal{D}}\left(E_{+}, z\right)=\omega_{\Delta}\left(\phi_{+}(E), \phi(z)\right)$ and $\omega_{\mathbb{D}}\left(E_{-}, z\right)=\omega_{\Delta}\left(\phi_{-}(E), \phi(z)\right)$. We shall use the sum of these two measures, and we thus define

$$
\omega_{\mathcal{D}}(E, z)=\omega_{\mathscr{D}}\left(E_{+}, z\right)+\omega_{\mathfrak{D}}\left(E_{-}, z\right) .
$$

We have

$$
\omega_{\mathcal{D}}\left(E_{+}, z\right)=\frac{1}{\pi} \int_{E} \frac{1-|\phi(z)|^{2}}{\left|\phi_{+}(t)-\phi(z)\right|^{2}} \sqrt{\frac{t^{2}}{t^{2}-1}} \frac{\mathrm{d} t}{t^{2}},
$$


and similarly for $\omega_{\mathbb{D}}\left(E_{-}, z\right)$. Furthermore, if $E=[\alpha, \beta]$ then $\lim _{z \rightarrow x} \omega_{D}(E, z)=1$ for $x \in(\alpha, \beta)$. We stress that the limit is 1 both when $z$ approaches $x \in(\alpha, \beta)$ from above and from below.

For each Borel set $E$ in $\partial \mathcal{D}, \omega_{\mathcal{D}}(E, \cdot)$ is a positive harmonic function in $\mathscr{D}$. We also see that, for example,

$$
\omega_{D}([r, r+2], 0) \leq \frac{\text { const }}{r^{2}},
$$

for $r \geq 1$, with some constant independent of $r$. For $r=1$ it is definitely true, and for $r>1$ we have

$$
\omega_{\mathcal{D}}\left([r, r+2]_{+}, 0\right)=\frac{1}{\pi} \int_{r}^{r+2} \frac{1}{\left|\phi_{+}(t)\right|^{2}} \sqrt{\frac{t^{2}}{t^{2}-1}} \frac{\mathrm{d} t}{t^{2}} \leq \mathrm{const} \int_{r}^{r+2} \frac{\mathrm{d} t}{t^{2}} \leq \frac{\text { const }}{r^{2}},
$$

since

$$
\frac{1}{\left|\phi_{+}(t)\right|^{2}} \sqrt{\frac{t^{2}}{t^{2}-1}}
$$

is bounded.

The proof of Theorem 5 follows the same lines as the proof of Theorem 2.2 of [13], where it was shown that the polynomials are not dense in the corresponding $L^{2}$-space, but now based on the proposition below.

Proposition 1. Let $a>0$. There exists a constant $C$, depending only on $a$, such that

$$
\int_{-\infty}^{\infty} \frac{\log ^{+}|p(x)|}{x^{2}+1} \mathrm{~d} x \leq C \int_{|x| \geq a} \frac{\log ^{+}|p(x)|}{x^{2}+1} \mathrm{~d} x
$$

for all polynomials $p$.

Proof. (This proof is inspired by the arguments in [9, Chapter VIII, Section A.3].) We may take $a=\frac{1}{2}$. Let $p$ be any polynomial and consider the function

$$
v(z)=\int_{-1 / 2}^{1 / 2} \log ^{+}|p(z+t)| \mathrm{d} t .
$$

This is a subharmonic function in the complex plane and satisfies $v(z) \leq o(|z|)$, as $z$ tends to infinity.

For $n \geq 1$ and $x \in[n, n+1]$, we find that

$$
v(x) \leq \int_{n-1 / 2}^{n+3 / 2} \log ^{+}|p(t)| \mathrm{d} t \equiv v_{n}
$$

and, similarly, that

$$
v(x) \leq \int_{-(n+3 / 2)}^{-(n-1 / 2)} \log ^{+}|p(t)| \mathrm{d} t \equiv v_{-n},
$$

for $n \geq 1$ and $x \in[-(n+1),-n]$.

From the definition of $v_{ \pm n}$, we obtain

$$
\frac{v_{n}}{n^{2}} \leq \mathrm{const} \int_{n-1 / 2}^{n+3 / 2} \frac{\log ^{+}|p(t)|}{t^{2}+1} \mathrm{~d} t, \quad n \geq 1,
$$


and

$$
\frac{v_{-n}}{n^{2}} \leq \mathrm{const} \int_{-(n+3 / 2)}^{-(n-1 / 2)} \frac{\log ^{+}|p(t)|}{t^{2}+1} \mathrm{~d} t, \quad n \geq 1 .
$$

We consider the function

$$
m(z)=\sum_{n=1}^{\infty}\left(v_{n} \omega_{D}\left(E_{n}, z\right)+v_{-n} \omega_{D}\left(-E_{n}, z\right)\right),
$$

where $E_{n}=\left[n-\frac{1}{2}, n+\frac{3}{2}\right]$ for $n>1$ and $E_{1}=\left[1, \frac{5}{2}\right]$. For $z=0$ we have, by (6) and the relations above involving $v_{ \pm n}$,

$$
\begin{aligned}
m(0) & \leq \text { const } \sum_{n=1}^{\infty} \frac{v_{n}+v_{-n}}{n^{2}} \\
& \leq \text { const } \sum_{n=1}^{\infty}\left(\int_{n-1 / 2}^{n+3 / 2}+\int_{-(n+3 / 2)}^{-(n-1 / 2)}\right) \frac{\log ^{+}|p(t)|}{t^{2}+1} \mathrm{~d} t \\
& \leq \text { const } \int_{|t| \geq 1 / 2} \frac{\log ^{+}|p(t)|}{t^{2}+1} \mathrm{~d} t .
\end{aligned}
$$

The function $m$ is the limit of an increasing sequence of harmonic functions in $\mathcal{D}$. By Harnack's theorem (see, for example, [14, p. 16]) this limit is either identically equal to $\infty$ or it is a harmonic function in $\mathscr{D}$. Since $m(0)$ converges, $m$ is harmonic. Hence, the function $v(z)-m(z)$ is subharmonic in $\mathcal{D}$ and satisfies $v(z)-m(z) \leq o(|z|)$ as $z$ tends to infinity. If $x>1$ then we choose an $n \geq 1$ such that $x \in(n, n+1]$. Then

$$
\lim _{z \rightarrow x} v_{n} \omega_{D}\left(E_{n}, z\right)=v_{n} \geq v(x)
$$

and, therefore,

$$
\limsup _{z \rightarrow x}(v(z)-m(z)) \leq 0,
$$

for all $x>1$. Similarly, we find that

$$
\limsup _{z \rightarrow x}(v(z)-m(z)) \leq 0,
$$

for all $x<-1$, and therefore all boundary values, except at \pm 1 , of $v(z)-m(z)$ are less than or equal to zero. At \pm 1 , we have

$$
\limsup _{z \rightarrow \pm 1}(v(z)-m(z)) \leq v_{ \pm 1}
$$

Lemma 3, below, implies that, for example, $v(z)-m(z) \leq v_{-1}+v_{1}$ in $\mathscr{D}$. In particular,

$$
\begin{aligned}
\int_{|t| \leq 1 / 2} \frac{\log ^{+}|p(t)|}{t^{2}+1} \mathrm{~d} t & \leq \int_{|t| \leq 1 / 2} \log ^{+}|p(t)| \mathrm{d} t \\
& =v(0) \\
& \leq m(0)+v_{-1}+v_{1} \\
& \leq \text { const } \int_{|t| \geq 1 / 2} \frac{\log ^{+}|p(t)|}{t^{2}+1} \mathrm{~d} t .
\end{aligned}
$$

This completes the proof. 
The following lemma is a slight variation on the maximum principle.

Lemma 3. Suppose that $u$ is subharmonic in $\mathscr{D}$, that $u(z) \leq o(|z|)$ as $|z|$ tends to infinity, and that $\lim \sup _{z \rightarrow x} u(z) \leq$ a for all $x \in \partial \mathscr{D}$. Then $u(z) \leq$ a throughout $\mathscr{D}$.

Proof. We let $Y_{\mathscr{D}}(z)=\operatorname{Im}\left(z^{2}-1\right)^{1 / 2}$, where we choose the square root that is positive and imaginary for $z \in(-1,1)$. This is a harmonic function in $\mathscr{D}$, with $Y_{\mathscr{D}}(x)=0$ for $x \in \partial \mathscr{D}$ and $Y_{\mathscr{D}}(z) \geq|\operatorname{Im} z|$ for $z \in \mathscr{D}$. (It is a so-called Phragmén-Lindelöf function (see, for example, [9, Chapter VIII, Section A.2])).

Let $\varepsilon>0$ be given, and consider the subharmonic function $u_{\varepsilon}(z)=u(z)-\varepsilon Y_{\mathscr{D}}(z)$ in $\mathscr{D}$. We shall verify that this function is bounded from above in $\mathcal{D}$. Then we apply an extended maximum principle (see, for example, [9, Chapter III, Section C]).

We put $S=\{x \geq 1, y \geq 0\}$. It is clear that $u_{\varepsilon}(z)=u(z)-\varepsilon Y_{\mathscr{D}}(z)$ is bounded from above on the boundaries of the four sectors $S,-S, \bar{S}$, and $-\bar{S}$. From a standard Phragmén-Lindelöf result, we then find that the function is bounded from above in all of these sectors (see, for example, [9, Chapter III, Section C]). It is also bounded from above in $\{|x| \leq 1\}$, so, in fact, it is bounded from above in $\mathcal{D}$.

We remark that a stronger form of Lemma 3 holds. From the assumptions that

$$
\limsup _{z \rightarrow x} u(z) \leq 0
$$

for all $x \in \partial \mathcal{D} \backslash\{ \pm 1\}$, and $\lim \sup _{z \rightarrow \pm 1} u(z) \leq a$, we are able to deduce that $u(z) \leq 0$ still holds throughout $\mathcal{D}$. This is because $u_{\varepsilon}(\psi(w))$ can be shown to be a subharmonic function that is bounded from above in the bounded domain $\Delta$. In that situation we can even exclude a sequence of points from the assumption on the boundary values.

\section{References}

[1] Berg, C. (1988). The cube of a normal distribution is indeterminate. Ann. Prob. 16, 910-913.

[2] Berg, C. (1995). Indeterminate moment problems and the theory of entire functions. J. Comput. Appl. Math. 65, 27-55.

[3] Berg, C. (2004). Correction to a paper by A. G. Pakes. J. Austral. Math. Soc. 76, 67-73.

[4] Borell, C. (1975). The Brunn-Minkowski inequality in Gauss space. Invent. Math. 30, 207-216.

[5] Borell, C. (2003). The Ehrhard inequality. C. R. Math. Acad. Sci. Paris 337, 663-666.

[6] Ehrhard, A. (1983). Symétrisation dans l'espace de Gauss. Math. Scand. 53, 281-301.

[7] Hoffmann-Jørgensen, J., Shepp, L. A. and Dudley, R. (1979). On the lower tail of Gaussian seminorms. Ann. Prob. 7, 319-342.

[8] Hörfelt, P. (2003). Geometric bounds on certain sublinear functionals of geometric Brownian motion. J. Appl. Prob. 40, 893-905.

[9] Koosis, P. (1988). The Logarithmic Integral. I (Camb. Studies Adv. Math. 12). Cambridge University Press.

[10] KreIn, M. G. (1945). On an extrapolation problem due to Kolmogorov. Dokl. Acad. Nauk. SSSR 46, $306-309$.

[11] NikeghbaLI, A. (2002). Moment problem for some convex functionals of Brownian motion and related problems. Working Paper 706, Université Paris VI.

[12] Pakes, A. G. (2001). Remarks on converse Carleman and Krein criteria for the classical moment problem. J. Austral. Math. Soc. 71, 81-104.

[13] Pedersen, H. (1998). On Krein's theorem for indeterminacy of the classical moment problem. J. Approx. Theory 95, 90-100.

[14] Ransford, T. (1995). Potential Theory in the Complex Plane (London Math. Soc. Student Texts 28). Cambridge University Press.

[15] Sudakov, V. N. ANd Cirel'son, B. S. (1978). Extremal properties of half-spaces for spherically invariant measures. J. Soviet Math. 9, 9-18.

[16] Yor, M. (1992). On some exponential functionals of Brownian motion. Adv. Appl. Prob. 24, 509-531. 